\title{
Urinotórax: una rara complicación de la nefrolitotomía percutánea: reporte de un caso
}

\author{
Urinothorax: rare complication of percutaneous nephrolithotomy. Case report
}

Javier Pérez-Palenzuela*, José G. Sosa-Martín, Kymani Pérez-García, Arturo Martínez-García y

José M. Díaz-Calderín

Servicio de Cirugía General, Hospital Clínico Quirúrgico Hermanos Ameijeiras, Universidad de Ciencias Médicas de la Habana, La Habana, Cuba

\begin{abstract}
Resumen
El urinotórax es una causa inusual de derrame pleural. Se describe un caso de urinotórax secundario a trauma del sistema urinario. Varón de 41 años que reingresa en el hospital a los 16 días de someterse a una nefrolitotomía percutánea derecha, con fiebre, disnea y dolor torácico. Se confirma por tomografía derrame pleural multitabicado, que se resolvió con tratamiento quirúrgico. Reingresa 21 días después con empiema recidivante y se diagnostica una fístula renopleural, que fue tratada con retoracotomía y colocación de catéter doble $J$ y sonda vesical. La sospecha diagnóstica y el manejo multidisciplinario permitieron resolver esta rara complicación.
\end{abstract}

Palabras clave: Urinotórax. Empiema. Nefrolitotomía percutánea.

\begin{abstract}
Urinothorax is an unusual cause of pleural effusion. To describe a case with urinothorax secondary to urinary system trauma. 41-year-old male readmitted to our hospital 16 days after a right percutaneous nephrolithotomy with fever, shortness of breath, chest pain and multiloculated pleural effusion confirmed by CT scan, resolved with surgical treatment. Readmitted twenty-one days later with recurrent empyema and diagnosis of reno-pleural fistula, treated with new thoracotomy and double $J$ and urinary catheter placement. The suspected diagnosis and the multidisciplinary approach allowed to solve this rare complication.
\end{abstract}

Key words: Urinothorax. Empyema. Percutaneous nephrolithotomy.

\section{Introducción}

El urinotórax se define como la presencia de orina en la cavidad pleural, lo que constituye una rara presentación de derrame pleural. Esta complicación fue reportada por primera vez por Corriere, et al. ${ }^{1}$ en 1968 , durante experimentos que incluían obstrucción ureteral en perros. Su estudio demostró una relación directa entre la hidronefrosis y el derrame pleural. Desde 1968 hasta la fecha se han reportado menos de 100 casos de urinotórax, lo que propició que los médicos tuvieran en cuenta con más regularidad este diagnóstico².

El conocimiento de esta complicación, el advenimiento de estudios imagenológicos de alta precisión

\section{Correspondencia:}

*Javier Pérez-Palenzuela

Vista Hermosa 602, Apto. 8

Entre Concepción y Santa Ana

Fecha de recepción: 05-01-2021

Cerro C.P. 10600, La Habana, Cuba

E-mail: jape122@gmail.com
Cir Cir. 2021;89(S2):17-21

Contents available at PubMed www.cirugiaycirujanos.com (http://creativecommons.org/licenses/by-nc-nd/4.0/). 
y el análisis bioquímico del líquido pleural han permitido establecer con frecuencia su diagnóstico precoz $^{3,4}$.

El objetivo de esta presentación es describir un caso de urinotórax como resultado de un proceder sobre el tracto urinario, teniendo en cuenta su poca frecuencia, a tal punto de ser el primer caso reportado en el servicio de litotricia del Hospital Clínico Quirúrgico Hermanos Ameijeiras.

\section{Caso clínico}

Varón de 41 años con antecedentes de diabetes mellitus tipo 2 y diagnóstico previo por imagenología de litiasis coraliforme en el grupo calicial superior del riñón derecho, de aproximadamente $23 \mathrm{~mm}$, con ectasia. Se le realiza nefrolitotomía percutánea derecha, con abordaje supracostal por encima de la $11 .{ }^{a}$ costiIla, con litofragmentación y extracción de cálculos, colocación de catéter nefro-pielo-ureteral por estenosis inflamatoria del cáliz superior y nefrostomía percutánea (Fig. 1).

Fue dado de alta a los 2 días y reingresó 16 días después por presentar un cuadro clínico de fiebre de $38{ }^{\circ} \mathrm{C}$, falta de aire y dolor torácico derecho. En la exploración física del sistema respiratorio se encuentran expansibilidad torácica disminuida, vibraciones vocales ausentes y murmullo vesicular abolido en la base pulmonar derecha, con una frecuencia respiratoria de 20 por minuto y una $\mathrm{SO}_{2}$ del $97 \%$, sin otras alteraciones. Se le realiza analítica sanguínea, que muestra leucograma de $12 \times 10^{9} /$, con desviación a la izquierda, cituria negativa y resto de la bioquímica sanguínea normal. En la radiografía de tórax se observa derrame pleural derecho (Fig. 2).

Se le realiza una tomografía computarizada (TC) de tórax que confirma el derrame pleural derecho de moderada cuantía, con múltiples tabiques y atelectasia del lóbulo inferior secundaria al derrame. Se discute el caso en el grupo de cirugía torácica y por los hallazgos clínicos e imagenológicos se interpreta como empiema pleural derecho, por lo que se realiza videotoracoscopia diagnóstica y terapéutica. Se encuentran múltiples adherencias firmes y se decide convertir el proceder a minitoracotomía, con drenaje de $600 \mathrm{ml}$ de líquido seroso turbio con múltiples esfacelos, y se realiza lavado de la cavidad torácica. El cultivo del líquido informa Staphylococcus aureus, por lo que se inicia tratamiento con vancomicina $(500 \mathrm{mg} / 6 \mathrm{~h})$. Se

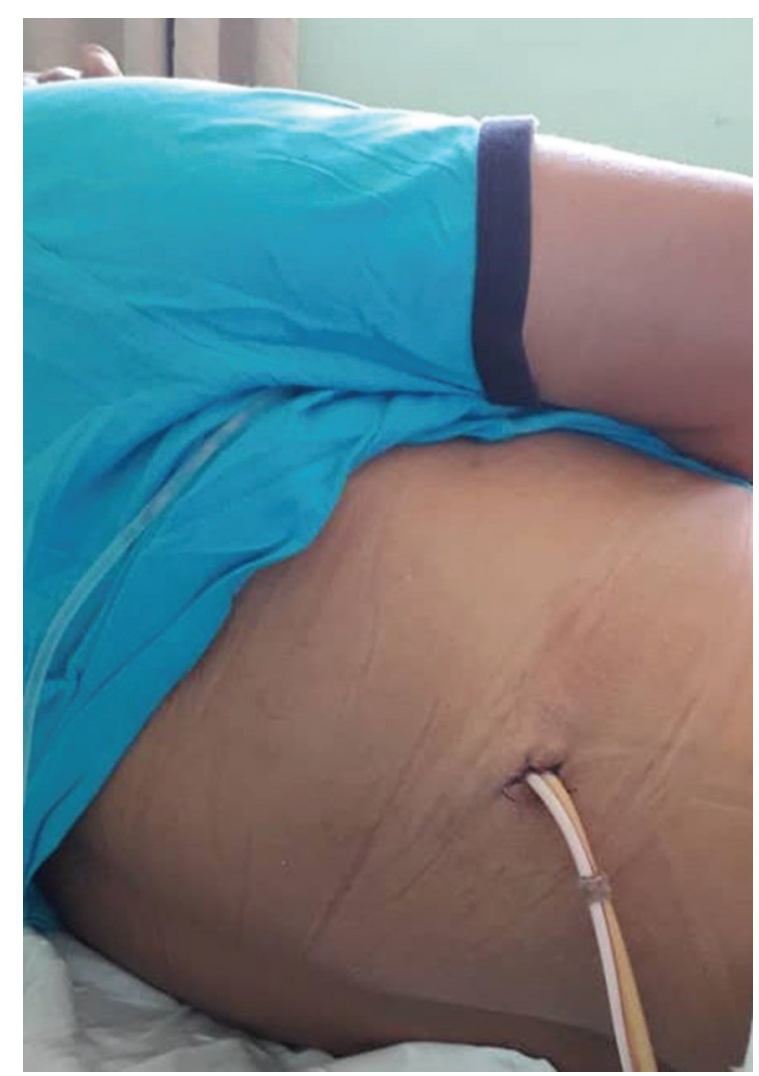

Figura 1. Catéter nefro-pielo-ureteral y nefrostomía percutánea tras la nefrolitotomía percutánea.

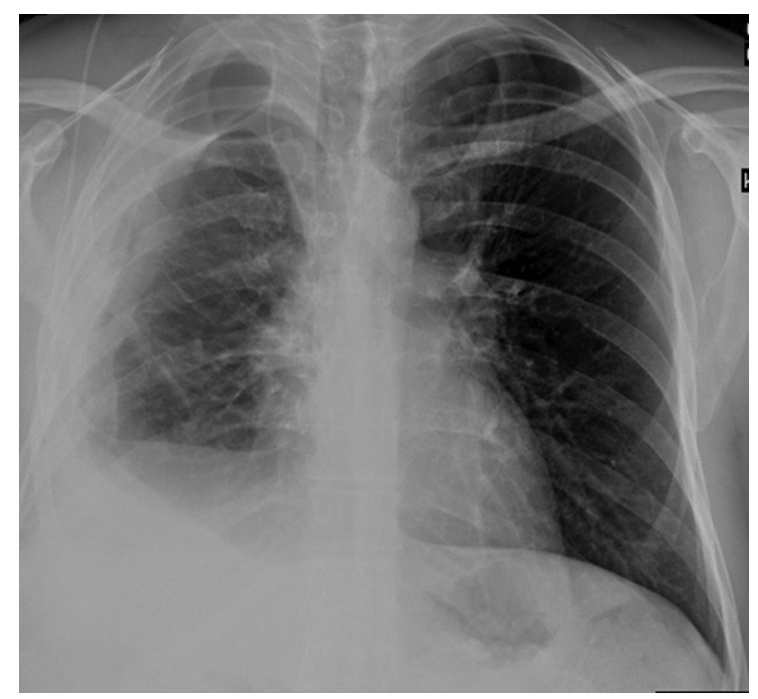

Figura 2. Radiografía de tórax posteroanterior de pie, que muestra un derrame pleural derecho de moderada cuantía.

mantiene un drenaje torácico hemático de alrededor de $200 \mathrm{ml} / \mathrm{día}$, sin burbujeo y con nefrostomía funcional, por lo que se decide, por parte del servicio 
de litotricia, retirar la sonda de nefrostomía. A los 7 días muestra mejoría clínica notable y drenaje escaso y seroso por la sonda de pleurostomía. La TC evolutiva informa reexpansión pulmonar sin derrame, por lo que se decide retirar la sonda pleural y dar el alta hospitalaria.

Tres semanas después reingresa en el servicio de cirugía por síndrome febril y derrame pleural derecho de moderada cuantía, confirmado por radiografía de tórax. Se le realiza ultrasonido torácico que confirma el diagnóstico, reportando además múltiples tabiques. En ese momento el paciente se encontraba asintomático, con hematocrito de 0.26 , leucograma de $15.4 \times$ $10^{9} / \mathrm{l}$ y creatinina de $65 \mu \mathrm{mol} / \mathrm{l}$.

Es llevado nuevamente al salón de operaciones, donde se le realiza una retoracotomía, encontrándose $500 \mathrm{ml}$ de líquido amarillento que en ese momento hace sospechar la presencia de orina. Además, durante la exploración se palpa un pequeño orificio fistuloso en el receso costofrénico posterior. Se colocan dos sondas pleurales al valorar la posibilidad de pleuroclisis terapéuticas (Fig. 3).

En el estudio bioquímico del líquido se cuantifica la creatinina en $66 \mu \mathrm{mol} / \mathrm{l}$ y la urea en $5.31 \mu \mathrm{mol} / \mathrm{l}$, con valores en sangre de $38 \mu \mathrm{mol} / / \mathrm{l}$ y $4.5 \mu \mathrm{mol} / \mathrm{l}$, respectivamente, lo cual confirmó el diagnóstico de urinotórax.

La TC contrastada de abdomen informa enfisema subcutáneo en la pared lumbar derecha, distorsión de la grasa perirrenal, sin líquido libre ni colecciones, irregularidad y afinamiento de la cortical a nivel del polo superior del riñón derecho y una imagen que impresiona ser un trayecto fistuloso (Fig. 4).

Es reevaluado por el servicio de litotricia y se le realiza una ureteropielografía retrógrada, con colocación de un catéter interno-externo e inyección de contraste yodado, con escape de orina por el cáliz superior (fístula renopleural), dejándose un catéter ureteral doble $\mathrm{J}$ y sonda vesical.

Evolutivamente, con el resultado del cultivo positivo para $S$. aureus y la persistencia de la fístula, se decide añadir pleuroclisis diarias con antibióticos intrapleurales (cloranfenicol), además de antibioticoterapia sistémica con linezolid (600 mg/12 h).

Una semana después se repite el estudio urinario contrastado y no se evidencia la persistencia de la fístula, por lo que se decide retirar la sonda vesical. Al día siguiente se constata un drenaje pleural mínimo, negatividad del cultivo del líquido pleural, reexpansión pulmonar por radiografía de tórax y ultrasonido

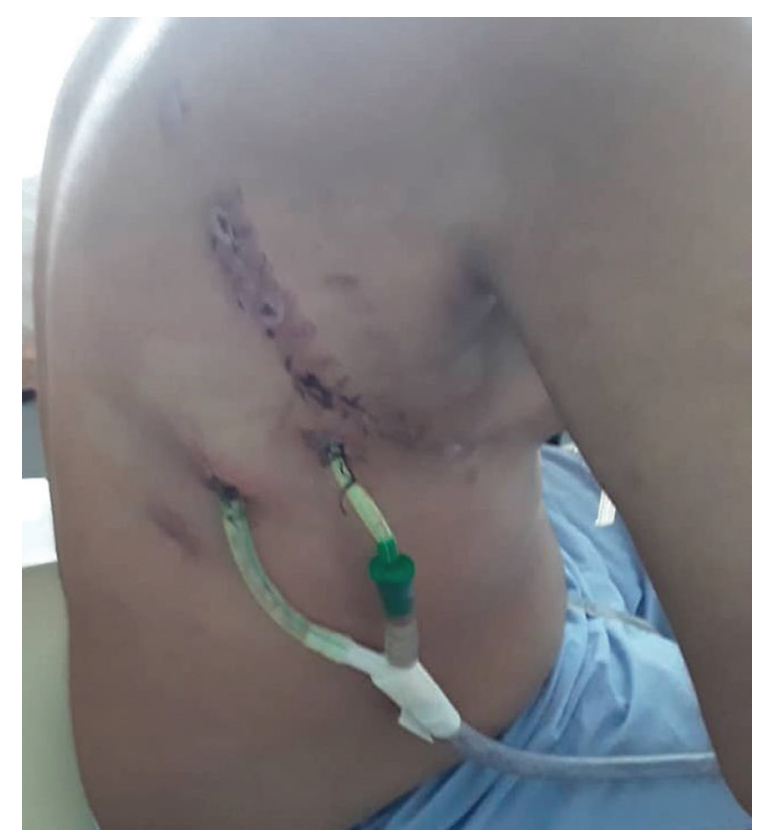

Figura 3. Paciente después de la reintervención, con dos sondas pleurales.

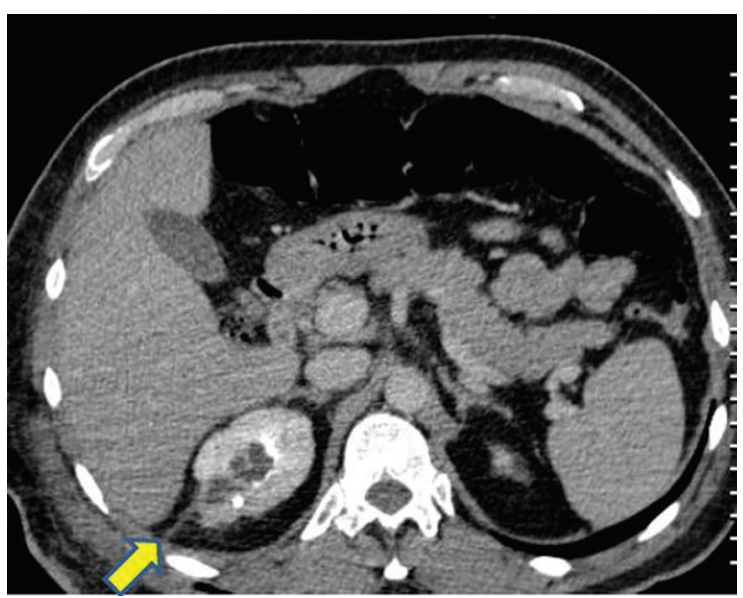

Figura 4. Tomografía computarizada con contraste intravenoso abdominal que muestra el trayecto fistuloso.

torácico que informa escaso líquido en la cavidad. Se decide retirar la sonda de pleurotomía y se da el alta médica por ambos servicios, con seguimiento en consulta. Seis semanas después, en el seguimiento por la consulta de litotricia, se realiza estudio urinario contrastado que no muestra alteraciones, por lo que se retira el catéter doble $\mathrm{J}$. A los 9 meses mantiene una evolución favorable en la consulta de cirugía, corroborada por la radiografía de tórax evolutiva (Fig. 5). 


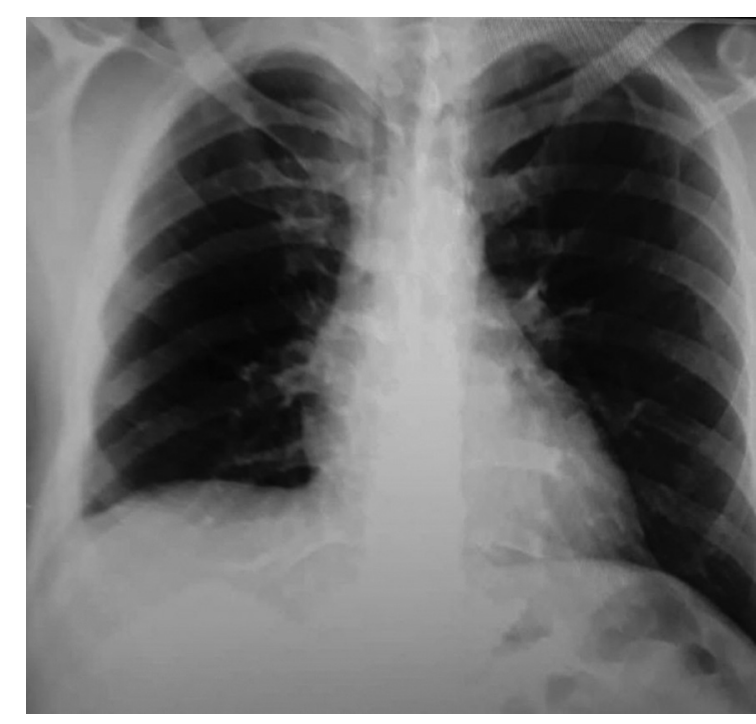

Figura 5. Radiografía a los 9 meses del alta médica.

\section{Discusión}

\section{Etiología}

Las causas de urinotórax pueden clasificarse en obstructivas 0 traumáticas ${ }^{5}$. La etiología más común es la extravasación de orina del tracto genitourinario, que lleva a la formación de un urinoma. Los urinomas se originan clásicamente de uropatías obstructivas, trauma o lesión iatrogénica del sistema urogenital. Otras causas de urinotórax se han reportado en el curso de malignidades genitourinarias, enfermedad metastásica, quistes renales, procesos inflamatorios retroperitoneales y embarazo. Dentro de la etiología traumática, las más comunes son las biopsias renales, la litotricia ureteroendoscópica, la nefrostomía percutánea y la nefrolitotomía percutánea ${ }^{6-8}$.

El mecanismo del tránsito de orina a través del diafragma a la cavidad pleural es debatido, pero predominan dos teorías. La primera es que el drenaje linfático diafragmático de orina resulta de un incremento de la presión en el retroperitoneo, típicamente secundario a un urinoma. La segunda teoría es que el paso de orina a la cavidad pleural se produce a través de un defecto anatómico en el diafragma, con frecuencia resultante de un trauma.

Dada la baja posición del diafragma, en conjunción con la muy posterior localización del receso costofrénico y el espacio pleural, es comprensible que se pueda atravesar el espacio pleural cuando se intenta el acceso al tracto urinario $0^{9,10}$. La mayoría de los casos de urinotórax son unilaterales y ocupan más de dos tercios del hemitórax, pero se han reportado raros casos de presentación bilateral ${ }^{6,11}$.

\section{Presentación}

El urinotórax puede ocurrir a cualquier edad, con una media de 45 años, y es más frecuente en el sexo masculino. La presentación suele ser aguda y se caracteriza por disnea, dolor torácico, dolor abdominal y disminución de la diuresis. En caso de existir uropatía obstructiva, el paciente puede estar febril y con dolor en el flanco. Los pacientes, además, pueden presentar taquicardia y taquipnea. La exploración física revela una disminución de los movimientos respiratorios, matidez a la percusión unilateral y ausencia de murmullo vesicular en el hemitórax afectado ${ }^{6}$.

\section{Imagenología}

Los estudios de imagen son útiles para el diagnóstico y existen múltiples modalidades disponibles. La radiografía simple de tórax puede mostrar un derrame homolateral, en ocasiones asociado con atelectasia pulmonar o desviación del mediastino ${ }^{9}$. El pielograma intravenoso es otra técnica para demostrar la extravasación de contraste del tracto genitourinario al espacio pleural. En su ausencia, se puede realizar una pielografía retrógrada, como se hizo en este paciente y permitió confirmar el diagnóstico. El ultrasonido y la TC con contraste intravenoso son útiles para determinar la afección genitourinaria que lleva a la formación del urinotórax, y en el caso de la última también puede ser útil para demostrar la fístula renopleural ${ }^{3,12}$.

\section{Diagnóstico}

El estudio del líquido pleural puede ser de utilidad; usualmente tendrá el mismo olor y el mismo color que la orina. Una relación de creatinina en líquido pleural/ suero $>1$ se considera de alta sospecha de urinotó$\operatorname{rax}^{13,14}$. En general es un trasudado paucicelular con baja concentración de glucosa y proteínas totales $<1 \mathrm{mg} / \mathrm{dl}^{7,13}$.

Los antecedentes clínicos, la bioquímica del fluido pleural y los estudios imagenológicos confirman el diagnóstico. 


\section{Tratamiento}

El reconocimiento temprano del urinotórax es muy importante para su tratamiento. Este va a depender de la causa que lo generó e incluye dos aspectos fundamentales: restablecer el flujo normal de orina y drenar la colección de esta en la cavidad pleural ${ }^{15}$. Ello requiere un enfoque multidisciplinario, con varios especialistas involucrados. En los casos en que sea posible, la toracocentesis puede realizarse con propósitos diagnósticos y terapéuticos ${ }^{2}$. En el paciente presentado, este paso se obvió al debutar con un derrame multitabicado que requirió tratamiento quirúrgico. En cualquier caso, el urinotórax persistirá mientras no se corrija la afección genitourinaria que lo generóz,16.

\section{Conclusiones}

Se debe tener una alta sospecha del urinotórax ante un paciente que se presenta con disnea, dolor torácico y antecedentes de un proceder invasivo sobre el tracto genitourinario. Es vital reconocer los síntomas y los signos de urinotórax precozmente en su evolución, para poder obtener los mejores resultados terapéuticos.

\section{Responsabilidades éticas}

Protección de personas y animales. Los autores declaran que para esta investigación no se han realizado experimentos en seres humanos ni en animales.

Confidencialidad de los datos. Los autores declaran que han seguido los protocolos de su centro de trabajo sobre la publicación de datos de pacientes.

Derecho a la privacidad y consentimiento informado. Los autores han obtenido el consentimiento informado de los pacientes y/o sujetos referidos en el artículo. Este documento obra en poder del autor de correspondencia.

\section{Conflicto de intereses}

Los autores declaran no tener ningún conflicto de intereses.

\section{Financiamiento}

No se requirió ningún tipo de financiamiento para la realización de este trabajo.

\section{Bibliografía}

1. Corriere JN, Miller WT, Murphy JJ. Hydronephrosis as a cause of pleural effusion. Radiology. 1968;90:79-84.

2. Dregoesc D, Kelley R, Lick D. An unusual case of urinothorax after percutaneous nephrostolithotomy. J Family Med Prim Care. 2019;8:2155-57.

3. Chandra A, Pathak A, Kapur A, Russia N, Bhasin N. Urinothorax: a rare cause of severe respiratory distress. Indian J Crit Care Med. 2014;18:320-2.

4. Wunderle K, Kim S, Chiovaro J. Urinothorax: a rare case of pleural effusion. J Gen Intern Med. 2017;32:1058-9.

5. García-Pachón E, Romero S. Urinothorax: a new approach. Curr Opin Pulm Med. 2006;12:259-63.

6. Toubes ME, Lama A, Ferreiro L, Golpe A, Álvarez-Dobaño JM, González-Barcala FJ, et al. Urinothorax: a systematic review. J Thorac Dis. 2017;9:1209-18

7. Austin A, Jogani SN, Brasher PB, Argula RG, Huggins JT, Chopra A. The urinothorax: a comprehensive review with case series. Am J Med Sci. 2017;354:44-53.

8. Lee TW, Jang HN, Cho HS, Choi SM, Choi BH, Bae E, et al. Urinothorax after ultrasonography-guided renal biopsy: a case report. BMC Nephrol. 2018;19:104.

9. Ranjan V, Agrawal S, Chipde SS, Dosi R. Urinothorax: a path, less travelled: case report and review of literature. J Nat Sci Biol Med. 2015:6:213-6.

10. Salcedo JR. Urinothorax: report of 4 cases and review of the literature. J Urol. 1986;135:805-8.

11. Maitra NU, Conn IG. A unique case of spontaneous bilateral renal subcapsular urinoma secondary to high pressure chronic retention of urine. Urol Case Rep. 2017;14:21-3.

12. Batura D, Haylock-vize P, Naji Y, Tennant R, Fawcett K. Management of iatrogenic urinothorax following ultrasound guided percutaneous nephrostomy. J Radiol Case Rep. 2014;8:34-40.

13. Stark DD, Shanes JG, Baron RL, Koch DD. Biochemical features of urinothorax. Arch Intern Med. 1982;142:1509-11.

14. Ryu JH, Tomássetti S, Maldonado $\mathrm{F}$. Update on uncommon pleural effusions. Respirology. 2011;16:238-43.

15. Bahjat AS, Sadeeq KJ, Tahir AMS, Mohammed AA. Urinothorax causing massive left side pleural effusion in a young lady; case report and literature review. Urol Case Rep. 2019;26:100957.

16. Kelley M, Spieler B, Rouse C, Karl B, Marshall R, Carbonella G. Urinothorax: a rare complication of percutaneous nephrostomy. Radiol Case Rep. 2019;14:729-33. 